\title{
Feasibility model for freight train insertion in one way - train schedule
}

\author{
Arrifah Ratna Sari ${ }^{1, *}$ and Ahmad Rusdiansyah ${ }^{1}$ \\ ${ }^{1}$ Industrial Engineering, Faculty of Industrial Technology, Institut Teknologi Sepuluh Nopember, \\ 60111 Surabaya, Indonesia
}

\begin{abstract}
In 2014, 94\% distribution in Indonesia used by truck mode. But in recent years, the train mode begun to be used as a distribution transport mode of replacement trucks. For shipments with a distance of 500-1500 km, rail mode is more suitable due to cheaper transportation costs and faster delivery times. But in Indonesia railway infrastructure is only used $56 \%$ of total capacity. Therefore the current logistical challenge is how to optimize the use of railways. This research tries to develop mathematical model for determining freight trains that avoid the ovetaking. The model developed is a train slot model that inserts a freight train between fixed trains. Because there is a lot of input schedules, it requires a long computation time. Therefore, this research also develops decision support tools using Visual Basic Application in Microsoft Excel. The result showed that minimum headway effect on the determination of additional freight trains and the optimum minimum headway is 6 minutes. With 6 minutes of minimum headway, the additional freight train are 15 trains. This additional train increase the carrying capacity of 900 TEUs / day. When compared with the initial condition, it means the addition of transport capacity is $21,43 \%$
\end{abstract}

\section{Introduction}

In logistics, transportation has a function as a product movement and product storage. The cost of transportation can affects about $60 \%$ of the total logistics costs. Transportation therefore plays a very important role in the success of supply chain management.

In $2014,94 \%$ distribution in Indonesia used by truck mode. For shipments with a distance of $500-1500 \mathrm{~km}$, suitable transportation mode is train due to the transport cost efficiency and logistics. For raw material commodities with large shipping volumes or end products with low per-unit values and time-insensitive are suitable for this mode.

In 2017 passenger train the presentation of realization reached 4,11\% form the target value. On the contrary, the realization decrease reaches $-10.83 \%$ for freight trains. Then, as in the Surabaya - Bojonegoro line only uses $56 \%$ of the railway capacity per day. Meanwhile, every year, the growth of freight transport volume is targeted to increase by $19 \%$ per year. Therefore it is necessary to increase the schedule of freight trains to reach the target that has been determined.

\footnotetext{
* Corresponding author: arrifahratnasari@gmail.com
} 
There are some previous studies related to train scheduling, research [1] try to determine the multi-line schedule by adding train slots with respect of time demands and uncertain destinations. The developed model using simulation-based iterative framework approach, it give the result of minimal operational cost. Research [2] aims to find the addition of freight train schedules on complex networks by route selection method. The research produces train and delivery schedules that can reduce delays. Research [3] aims to scheduling extra freight train on railway network that can minimize the delay and find the optimum route to avoid the crossing between consecutive trains.

\section{Problem Description}

This research use [1] as a reference in the concept of timeslot. This concept used the schedule of passenger trains that have fixed characteristics. When the demand change, the output of the train schedule will be revised and developed with iterative procedures until the criteria are met or convergence is reached. The aims in this research is to construct mathematical models and designing decision-making tools to determine the numbers of freight trains that can be added with avoid the overtake. By using Visual Basic for Application in Microsoft Excel it can process large data automatically in accordance with the ordered programming languages. Excel macros also a user interface that is easy to understand and easy to operate as a tool for determining the policy later.

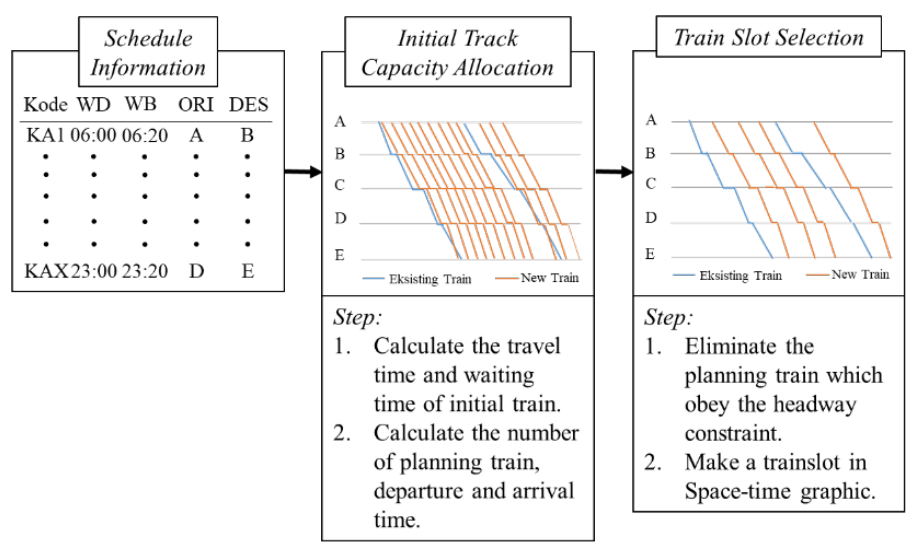

Fig. 1. Development of Train Slot Selection

Figure 1 describes an overview of the timetable development techniques used in this study. Starting from the 1 st plot is an information of the existing train schedule of both passenger and freight trains on the SBY - SMG line for each large and small station. In plot 2 is the step of forming additional train plans with respect to the existing schedule and minimum headway. In Plot 3 there is an additional train elimination plan that does not violate the specified boundary.

\section{Model and Algorithm}

The following is list of notations which are used in the model.

Subscripts:

$$
\begin{array}{ll}
u & =\text { index of reference train } \\
v & =\text { index of terminal } \\
x & =\text { index of additional train plan }
\end{array}
$$


$y=$ index of existing freight train to be inserted

Parameter:

$a_{u} \quad=$ reference train to- $u$, where $u=1$

$s_{v} \quad=$ terminal to- $v$, where $\forall v \in \widehat{V}$

$c_{x} \quad=$ additional train plan to- $x$, where $\forall x \in \hat{X}$

$e_{y} \quad=$ existing freight train to- $y$ to be inserted, where $\forall y \in \hat{Y}$

$\widehat{V} \quad=$ Number of terminal

$\hat{Y} \quad=$ Number of existing freight train to be inserted

$\hat{Z} \quad=$ Number of valid train

$\mathrm{h}=$ minimum headway

$M_{\left(c_{x}\right)\left(s_{v}\right)}=$ Value of additional train plan $x$ at terminal $v,\{0,1\}$

$M_{\left(c_{x}\right)} \quad=$ Value of additional train plan $x,\{0,1\}$

$W D_{\left(a_{u}\right)\left(s_{v}\right)}=$ Arrival time of reference train $a_{u}$ at terminal $s_{v}$

$W B_{\left(a_{u}\right)\left(s_{v}\right)}=$ Departure time of reference train $a_{u}$ at terminal $s_{v}$

$W D_{\left(e_{y}\right)\left(s_{v}\right)}=$ Arrival time of existing train $e_{y}$ at terminal $s_{v}$

$W B_{\left(e_{y}\right)\left(s_{v}\right)}=$ Departure time of existing train $e_{y}$ at terminal $s_{v}$

Decision Variable:

$W P_{\left(a_{u}\right)\left(s_{v}, s_{v+1}\right)} \quad=$ Travel time of reference train $a_{u}$ from terminal $s_{v}$ to $s_{v+1}$

$W T_{\left(a_{u}\right)\left(s_{v}\right)} \quad=$ Waiting time of reference train $a_{u}$ at terminal $s_{v}$

$D_{\left(e_{y}, e_{y+1}\right)\left(s_{v}\right)} \quad=$ Number of additional train plan between existing trains $y$ with $y+1$

$\hat{X} \quad=$ Number of additional train plan form existing train $e_{y}$ until $e_{\hat{Y}}$

$W B_{\left(c_{x}\right)\left(e_{y}, e_{y+1}\right)\left(s_{v}\right)}=$ Departure time of additional train plan $x$ between existing trains $y$ with train $y+1$ at terminal $v$

$W D_{\left(c_{x}\right)\left(e_{y}, e_{y+1}\right)\left(s_{v}\right)}=$ Arrival time of additional train plan $x$ between existing trains $y$ with train $y+1$ at terminal $v$

$\widehat{M} \quad$ = Number of valid train that doesn't violate minimum headway boundary

STEP 1: Calculate the travel time of reference train $a_{u}$ at every terminal $s_{v}$

Objective Function:

Min $W P_{\left(a_{u}\right)\left(s_{v}, s_{v+1}\right)}$

Subject To:

$W P_{\left(a_{u}\right)\left(s_{v}, s_{v+1}\right)}=\frac{\left(W D_{\left(e_{1}\right)\left(s_{v+1}\right)}-W B_{\left(e_{1}\right)\left(s_{v}\right)}\right)+\cdots+\left(W D_{\left(e_{\widehat{Y}}\right)\left(s_{v+1}\right)}-W B\left(e_{\widehat{Y}}\right)\left(s_{v}\right)\right)}{\hat{Y}} ; a=1 ; \forall v \in \hat{V} ;$

$\forall y \in \hat{Y}$

STEP 2: Calculate the waiting time of reference train $a_{u}$ at every terminal $s_{v}$

Objective Function:

Min $W T_{\left(a_{u}\right)\left(s_{v}\right)}$

Subject To:

$W T_{\left(a_{u}\right)\left(s_{v}\right)}=\frac{\left(W B_{\left(e_{1}\right)\left(s_{v}\right)}-W D_{\left(e_{1}\right)\left(s_{v}\right)}\right)+\cdots+\left(W B\left(e_{\widehat{Y}}\right)\left(s_{v}\right)^{-W D}\left(e_{\widehat{Y}}\right)\left(s_{v}\right)\right)}{\hat{Y}} ; u=1 ; v=2,3, \ldots, \hat{V} ;$

$\forall y \in \hat{Y}$

Repeat step 1 and 2 until the travel time and waiting time of reference train $a_{u}$ is obtained for each terminal $s_{v}$, where $\forall v \in \widehat{V}$.

STEP 3: Calculate the number of additional train plan between existing trains $e_{y}$ with $e_{y+1}$ at reference terminal.

Objective Function:

$\operatorname{Max} D_{\left(e_{y}, e_{y+1}\right)\left(s_{v}\right)}$ 
Subject To:

$W B_{\left(e_{y}\right)\left(s_{v}\right)}+D_{\left(e_{y}, e_{y+1}\right)\left(s_{v}\right)} x h<W B_{\left(e_{y+1}\right)\left(s_{v}\right)} \quad v=1 ; \forall y \in \hat{Y}$

$D_{\left(e_{y}, e_{y+1}\right)\left(s_{v}\right)} \geq 1$, And integer

STEP 4: Calculate the number of additional train plan from existing train $e_{y}$ until $e_{\widehat{Y}}$

Objective Function:

$\operatorname{Max} \hat{X}$

Subject To:

$\hat{X}=D_{\left(e_{y}, e_{y+1}\right)\left(s_{v}\right)}+D_{\left(e_{y+1}, e_{y+2}\right)\left(s_{v}\right)}+\cdots+D_{\left(e_{\widehat{Y}-1}, e_{\widehat{Y}}\right)\left(s_{v}\right)} v=1 ; \forall y \in \hat{Y}$

$\hat{X} \geq 1$, And integer

STEP 5: Calculate departure time of additional train plan $c_{x}$ at reference terminal

Objective Function:

Min $W B_{\left(c_{x}\right)\left(e_{y}, e_{y+1}\right)\left(s_{v}\right)}$

Subject To:

$W B_{\left(c_{x}\right)\left(e_{y}, e_{y+1}\right)\left(s_{v}\right)}=W B_{\left(e_{y}\right)\left(s_{v}\right)}+(x) .(h) \quad v=1 ; y \in \hat{Y} ; \forall x \in \hat{X}$

STEP 6: Calculate arrival time of additional train plan $c_{x}$ at each terminal $s_{v}$

Objective Function:

Min $W D_{\left(c_{x}\right)\left(e_{y}, e_{y+1}\right)\left(s_{v}\right)}$

Subject To:

$W D_{\left(c_{x}\right)\left(e_{y}, e_{y+1}\right)\left(s_{v}\right)}=W B_{\left(c_{x}\right)\left(e_{y}, e_{y+1}\right)\left(s_{v-1}\right)}+W P_{\left(a_{u}\right)\left(s_{v-1}, s_{v}\right)}$

$u=1 ; v=2,3, \ldots, \hat{V} ; \forall y \in \hat{Y} ; \forall x \widehat{X}$

STEP 7: Calculate departure time of additional train plan $c_{x}$ at each terminal $\boldsymbol{s}_{v}$

Objective Function:

Min $W B_{\left(c_{x}\right)\left(e_{y}, e_{y+1}\right)\left(s_{v}\right)}$

Subject To:

$W B_{\left(c_{x}\right)\left(e_{y}, e_{y+1}\right)\left(s_{v}\right)}=W D_{\left(c_{x}\right)\left(e_{y}, e_{y+1}\right)\left(s_{v}\right)}+W T_{\left(a_{u}\right)\left(s_{v}\right)} ; u=1 ; v=2,3, \ldots, \widehat{V} ; \forall y \in \hat{Y} ; \forall x \in$ $\hat{X}$

Repeat step 5 and 6 until the departure time and arrival time for each additional train plan $c_{x}$ is obtained at every terminal $s_{v}$, where $\forall v \in \widehat{V}$.

STEP 8: Evaluate schedule of additional train plan according to minimum headway's boundary

$\begin{array}{ll}W D_{\left(e_{y}\right)\left(s_{v}\right)}+h \leq W D_{\left(c_{x}\right)\left(e_{y}, e_{y+1}\right)\left(s_{v}\right)} & \forall v \in \hat{V} ; \forall y \in \hat{Y} ; \forall x \in \hat{X} \\ W D_{\left(c_{x}\right)\left(e_{y}, e_{y+1}\right)\left(s_{v}\right)} \leq W D_{\left(e_{y+1}\right)\left(s_{v}\right)}-h & \forall v \in \hat{V} ; \forall y \in \hat{Y} ; \forall x \in \hat{X} \\ W B_{\left(e_{y}\right)\left(s_{v}\right)}+h \leq W B_{\left(c_{x}\right)\left(e_{y}, e_{y+1}\right)\left(s_{v}\right)} & \forall v \in \hat{V} ; \forall y \in \hat{Y} ; \forall x \in \hat{X} \\ W B_{\left(c_{x}\right)\left(e_{y}, e_{y+1}\right)\left(s_{v}\right)} \leq W B_{\left(e_{y+1}\right)\left(s_{v}\right)}-h & \forall v \in \hat{V} ; \forall y \in \hat{Y} ; \forall x \in \hat{X}\end{array}$

STEP 9: Calculate the number of valid train from existing train $e_{y}$ until train $e_{\widehat{Y}}$

Decision Variable:

$M_{c_{x}\left(W D_{\left(e_{y}\right)\left(s_{v}\right)}\right)} \begin{cases}1 & \text { If Constraint } 17 \text { Satisfy } \\ 0 & \text { Otherwise }\end{cases}$

$M_{c_{x}\left(W D_{\left(e_{y+1}\right)\left(s_{v}\right)}\right)} \begin{cases}1 & \text { If Constraint } 18 \text { Satisfy } \\ 0 & \text { Otherwise }\end{cases}$

$M_{c_{x}\left(W B_{\left(e_{y}\right)\left(s_{v}\right)}\right)} \begin{cases}1 & \text { If Constraint } 19 \text { Satisfy } \\ 0 & \text { Otherwise }\end{cases}$

$M_{c_{x}\left(W B_{\left(e_{y+1}\right)\left(s_{v}\right)}\right)} \begin{cases}1 & \text { If Constraint } 20 \text { Satisfy } \\ 0 & \text { Otherwise }\end{cases}$

Objective:

$\operatorname{Max} \widehat{M}$

Subject to: 


$$
\begin{aligned}
& M_{\left(c_{x}\right)\left(s_{v}\right)}=\left(M_{c_{x}\left(W D_{\left(e_{y}\right)\left(s_{v}\right)}\right)}\right)\left(M_{c_{x}\left(W D_{\left(e_{y+1}\right)\left(s_{v}\right)}\right)}\right)\left(M_{c_{x}\left(W B_{\left(e_{y}\right)\left(s_{v}\right)}\right)}\right)\left(M_{c_{x}\left(W B_{\left(e_{y+1}\right)\left(s_{v}\right)}\right)}\right) \\
& \forall v \in \widehat{V} ; \forall x \in \widehat{X} ; M_{x\left(s_{v}\right)}=\{0,1\} \\
& M_{\left(c_{x}\right)}=\left(M_{c_{x}\left(s_{v}\right)}\right)\left(M_{c_{x}\left(s_{v+1}\right)}\right)\left(M_{c_{x}\left(s_{v+2}\right)}\right) \ldots\left(M_{c_{x}\left(s_{\widehat{V}}\right)}\right) \quad \forall v \in \widehat{V} ; \forall x \in \widehat{X} ; M_{x}=\{0,1\} \\
& \widehat{M}=\left(M_{c_{x}}\right)+\left(M_{c_{x+1}}\right)+\left(M_{c_{x+2}}\right)+\cdots+\left(M_{c_{\widehat{X}}}\right) \\
& \widehat{M} \geq 1, \text { dan integer }
\end{aligned}
$$

\section{Numerical Experiment and Result}

The data used to calculate the mathematical model is the small data (iteration to 1) for each steps in the mathematical model. Table 1 is the summary result of steps 3 to 7 . KA $2503\left(e_{1}\right)$ and KA $2507\left(e_{2}\right)$ is the input of the existing schedule. Step 3 is to calculate the number of additional train plans between existing trains $e_{1}$ with $e_{2}$, there are 6 train plans. Step 4 is to calculate the number of additional train total plan that is from the existing train $e_{1}$ to $e_{20}$ there are 284 trains. Step $5-7$ is calculating the departure time and the arrival time of the train plan at each station. For example at Mesigit station, train plan $1\left(e_{1}\right)$ arrives at 00:18:00 and departs at 00:20:00.

Table 1 Departure and Arrival Time Planning Train at Terminal 1-4

\begin{tabular}{|l|r|r|r|r|r|r|r|r|}
\hline Terminal & \multicolumn{2}{|c|}{ KALIMAS } & \multicolumn{2}{c|}{ MESIGIT } & \multicolumn{2}{c|}{ PS TURI (SBI) } & \multicolumn{2}{c|}{ TANDES (TES) } \\
\hline Time & AT & DT & AT & DT & AT & DT & AT & DT \\
\hline KA 2503 & $00: 05: 00$ & $00: 05: 00$ & $00: 12: 00$ & $00: 14: 00$ & $00: 18: 00$ & $00: 23: 00$ & $00: 32: 00$ & $00: 32: 00$ \\
\hline Plan 1 & & $00: 11: 00$ & $00: 18: 00$ & $00: 20: 00$ & $00: 24: 00$ & $00: 24: 00$ & $00: 33: 00$ & $00: 33: 00$ \\
\hline Plan 2 & & $00: 17: 00$ & $00: 24: 00$ & $00: 26: 00$ & $00: 30: 00$ & $00: 30: 00$ & $00: 39: 00$ & $00: 39: 00$ \\
\hline Plan 3 & & $00: 23: 00$ & $00: 30: 00$ & $00: 32: 00$ & $00: 36: 00$ & $00: 36: 00$ & $00: 45: 00$ & $00: 45: 00$ \\
\hline Plan 4 & & $00: 29: 00$ & $00: 36: 00$ & $00: 38: 00$ & $00: 42: 00$ & $00: 42: 00$ & $00: 51: 00$ & $00: 51: 00$ \\
\hline Plan 5 & & $00: 35: 00$ & $00: 42: 00$ & $00: 44: 00$ & $00: 48: 00$ & $00: 48: 00$ & $00: 57: 00$ & $00: 57: 00$ \\
\hline Plan 6 & & $00: 41: 00$ & $00: 48: 00$ & $00: 50: 00$ & $00: 54: 00$ & $00: 54: 00$ & $01: 03: 00$ & $01: 03: 00$ \\
\hline KA 2507 & $00: 50: 00$ & $00: 50: 00$ & $00: 57: 00$ & $00: 59: 00$ & $01: 03: 00$ & $01: 06: 00$ & $01: 15: 00$ & $01: 15: 00$ \\
\hline
\end{tabular}

Table 2 is an example of the calculation result for the determination of the value $M_{c_{1}\left(W D_{(e y)(v)}\right)}$ train plan 1 to existing trains 1 and 2 at stations 2 and 3 . In the Additional Train 1 plan at station 1 meet the boundary, but at station 3 violates the boundary so train

\begin{tabular}{|c|c|c|c|c|c|c|}
\hline \multirow{3}{*}{ Train Code } & \multicolumn{6}{|c|}{ Result (Time Conversion) } \\
\hline & \multicolumn{2}{|c|}{ Kalimas } & \multicolumn{2}{|c|}{ Mesigit } & \multicolumn{2}{|c|}{ Pasar Turi } \\
\hline & WD & WB & WD & WB & WD & WB \\
\hline KA 2503 & $00: 05: 00$ & $00: 05: 00$ & $00: 12: 00$ & $00: 14: 00$ & $00: 18: 00$ & $00: 23: 00$ \\
\hline$M_{c_{1}\left(W D_{\left(e_{\gamma}\right)(v)}\right)}$ & 1 & 1 & 1 & 1 & 1 & 0 \\
\hline $\mathrm{C} 1$ & 00:11:00 & 00:11:00 & 00:18:00 & 00:20:00 & 00:24:00 & 00:24:00 \\
\hline$M_{c_{1}\left(W D_{\left(e_{y+1}\right)(v)}\right)}$ & 1 & 1 & 1 & 1 & 1 & 1 \\
\hline KA 2507 & $00: 50: 00$ & $00: 50: 00$ & 00:57:00 & $00: 59: 00$ & 01:03:00 & 01:06:00 \\
\hline
\end{tabular}
plan 1 is invalid $\left(M_{c_{1}}=0\right)$. After an evaluation of the limits for all additional train plans, then calculate the total of additional train plans as many as 21 trains.

$$
\begin{aligned}
& M_{c_{1}\left(s_{1}\right)}=\left(M_{c_{1}\left(W D_{(e y)(v)}\right)}\right)\left(M_{c_{1}\left(W D_{\left(e_{y+1}\right)(v)}\right)}\right)\left(M_{c_{1}\left(W B_{(e y)}\right)}\right)\left(M_{c_{1}\left(W B_{\left(e_{y+1}\right)(v)}\right)}\right)=(1)(1)(1)(1)=1 \\
& M_{c_{1}}=\left(M_{c_{1}\left(s_{v}\right)}\right)\left(M_{c_{1}\left(s_{v+1}\right)}\right)\left(M_{c_{1}\left(s_{v+2}\right)}\right) \ldots\left(M_{c_{1}\left(s_{\widehat{V}}\right)}\right)=(1)(1)(0) \ldots(1)=0 \\
& \widehat{M}=\left(M_{c_{1}}\right)+\left(M_{c_{2}}\right)+\left(M_{c_{3}}\right)+\cdots+\left(M_{c_{284}}\right)=(0)+(1)+(0)+\cdots+(0)=21
\end{aligned}
$$

Table 2 Evaluation of Minimum Headway's Boundary 


\begin{tabular}{|l|l|l|l|}
\hline & Satisfy & Satisfy & Unsatisfied \\
\hline
\end{tabular}

Figure 2 is a graph of time space with minimum headway 6 minutes. Where with 6 minute headway got 15 additional trains. Y axis is the terminal along SBY Kalimas to SMG Poncol. While the $\mathrm{X}$ axis is time for 24 hours. Therefore the graph is said to be a space-time graph because it contains time and place. The graph with the orange arch is the graph of the existing train. While the graph with the blue arch is an additional plan train that meets the limits of min headway.

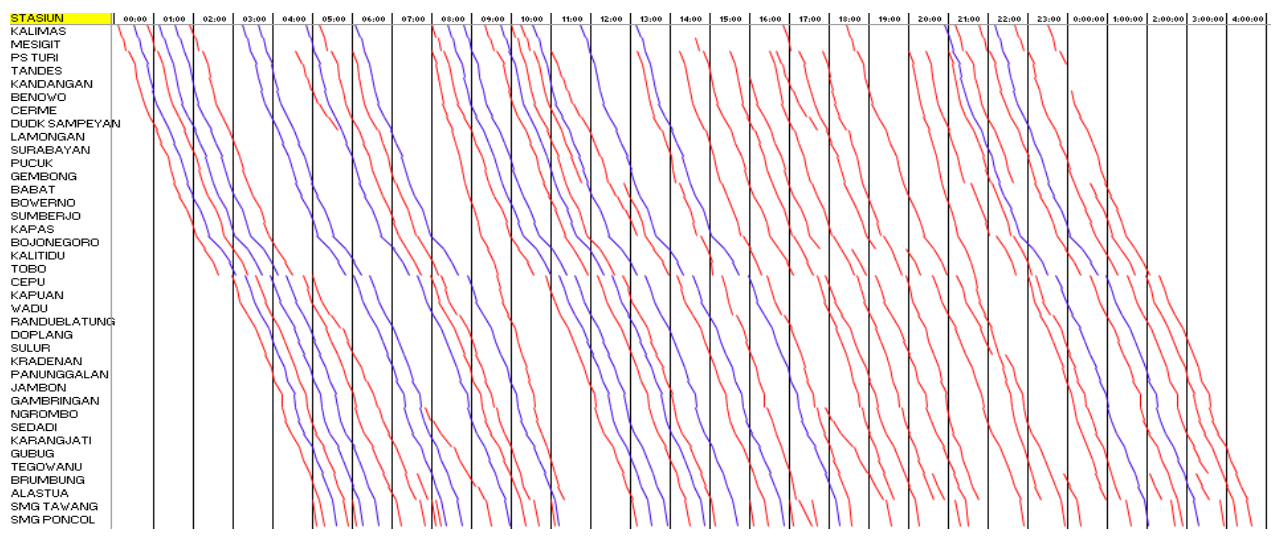

Fig. 2. Graphic Time Space with 6 Minutes Minimum Headway

\section{Conclusion}

This research produce mathematical model and the design of decision making tools for the determination of freight trains that can be added from the existing schedule. It can be concluded by using a minimum of 6 minutes headway can be produced by adding 15 freight trains. This means an increase in the addition of trains by $21,43 \%$ of the total number of existing trains. For further research, model can be developed by calculating station capacity, signalling system and blocking problem.

\section{References}

1. Kuo, A., Miller-Hooks, E., \& Mahmassani, H. S. Transportation Research Part E: Logistics and Transportation Review, 46, 6 (2010)

2. Mu, S., \& Dessouky, M. Scheduling freight trains traveling on complex networks. Transportation Research Part B: Methodological, 45, 7 (2011)

3. Cacchiani, V., Caprara, A., Toth, P., Scheduling Extra Freight Trains on Railway Networks, Transportation Research Part B, 44, 215-231 (2010) 